\title{
Multiple Nonauditory Cortical Regions Innervate the Auditory Midbrain
}

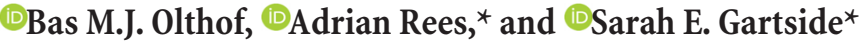 \\ Institute of Neuroscience, Newcastle University, Newcastle upon Tyne NE2 4HH, United Kingdom
}

Our perceptual experience of sound depends on the integration of multiple sensory and cognitive domains, however the networks subserving this integration are unclear. Connections linking different cortical domains have been described, but we do not know the extent to which connections also exist between multiple cortical domains and subcortical structures. Retrograde tracing in adult male rats (Rattus norvegicus) revealed that the inferior colliculus, the auditory midbrain, receives dense descending projections not only, as previously established, from the auditory cortex, but also from the visual, somatosensory, motor, and prefrontal cortices. While all these descending connections are bilateral, those from sensory areas show a more pronounced ipsilateral dominance than those from motor and prefrontal cortices. Injections of anterograde tracers into the cortical areas identified by retrograde tracing confirmed those findings and revealed cortical fibers terminating in all three subdivisions of the inferior colliculus. Immunolabeling showed that cortical terminals target both GABAergic inhibitory, and putative glutamatergic excitatory neurons. These findings demonstrate that auditory perception and behavior are served by a network that includes extensive descending connections to the midbrain from sensory, behavioral, and executive cortices.

Key words: cortex; inferior colliculus; motor; prefrontal; somatosensory; visual

\section{Significance Statement}

Making sense of what we hear depends not only on the analysis of sound, but also on information from other senses together with the brain's predictions about the properties and significance of the sound. Previous work suggested that this interplay between the senses and the predictions from higher cognitive centers occurs within the cerebral cortex. By tracing neural connections in rat, we show that the inferior colliculus, the subcortical, midbrain center for hearing, receives extensive connections from areas of the cerebral cortex concerned with vision, touch, movement, and cognitive function, in addition to areas representing hearing. These findings demonstrate that wide-ranging cortical feedback operates at an earlier stage of the hearing pathway than previously recognized.

\section{Introduction}

Sensory processing has traditionally been viewed as the centripetal flow of information from a sense organ to a modality-specific region of the cerebral cortex. This view is now being re-evaluated on two grounds. First, evidence for extensive feedback connections from higher levels to earlier stages in the processing hierar-

Received June 19, 2019; revised Aug. 2, 2019; accepted Aug. 21, 2019.

Author contributions: B.M.J.O., A.R., and S.E.G. designed research; B.M.J.O., A.R., and S.E.G. performed research; B.M.J.O. analyzed data; B.M.J.O., A.R., and S.E.G. wrote the first draft of the paper; B.M.J.O., A.R., and S.E.G. edited the paper; B.M.J.O., A.R., and S.E.G. wrote the paper.

This work was supported by Biotechnology and Biological Sciences Research Council Grant BB/P003249/1 to A.R. and S.E.G. We thank the Newcastle University Biolmaging Unit for support and assistance in performing the microscopy; Andrew Trevelyan for advice on tracer injection; and Michael Schmid for comments on an earlier version of the manuscript.

The authors declare no competing financial interests.

*A.R. and S.E.G. contributed equally to this work as joint senior authors.

Correspondence should be addressed to Bas M.J. Olthof at bas.oltho@@ncl.ac.uk.

https://doi.org/10.1523/JNEUROSCI.1436-19.2019

Copyright $\odot 2019$ the authors chy demonstrates the flow of information is not one way. Such top-down processing is in keeping with a growing appreciation that perception involves the interaction between predictions or expectations based on prior experience and incoming sensory information (Rao and Ballard, 1999; Friston, 2005; Bastos et al., 2012). A second challenge to the traditional view of sensory processing is recent evidence that areas of cortex previously considered to be exclusively unimodal, including so-called primary regions, can respond to stimuli of other modalities (Kayser and Logothetis, 2007).

Top-down and cross-modal sensory interactions have been most extensively explored in the cerebral cortex, but the organization of the auditory system, with its many subcortical centers, means that such interactions could potentially occur much earlier in the pathway. The inferior colliculus (IC), the midbrain auditory center, receives extensive top-down input from the auditory cortex (AC) (Diamond et al., 1969; Beyerl, 1978; Andersen et al., 1980; Saldaña et al., 1996; Winer et al., 1998; Budinger et al., 
2000; Bajo and Moore, 2005; Coomes et al., 2005; Bajo et al., 2007). Cortico-collicular projections to the dorsal and lateral cortices of the IC (ICD and ICL, respectively) are known to be particularly dense, but connections to the central nucleus (ICC), the main recipient of afferent input from lower level brainstem centers, have also been reported in rat (Feliciano and Potashner, 1995; Saldaña et al., 1996) and other species (Budinger et al., 2000; Bajo et al., 2007). These cortico-collicular connections mediate both short- and long-term plasticity that influences the response properties of neurons in the IC to sounds (Gao and Suga, 2000; Suga and Ma, 2003; Bajo et al., 2010; Bajo and King, 2013).

While research on cortico-collicular connections has focused almost exclusively on those originating from the AC, an earlier anatomical study (Cooper and Young, 1976), conducted before the advent of modern tracers, suggested that inputs to the IC might originate from more diverse cortical sources. Cooper and Young (1976) found degenerating fibers in the IC following lesions to the visual, "somaesthetic" (somatosensory) and motor cortices, suggestive of cortico-collicular inputs from these nonauditory cortices. Surprisingly, despite this evidence, the intriguing possibility that the IC receives extensive inputs from cortical areas other than those subserving audition has not been addressed systematically. The concept of cortical feedback from nonauditory regions to the IC would be congruent with evidence for nonauditory feedforward sensory inputs that terminate in the cortical subdivisions of the IC. These include connections from the somatosensory dorsal column and spinal trigeminal nuclei to ICL (Aitkin et al., 1978, 1981; Jain and Shore, 2006), and visual inputs from the retina (Itaya and Van Hoesen, 1982; Yamauchi and Yamadori, 1982) and sources in the brainstem, including the superior colliculus (Adams, 1980; Coleman and Clerici, 1987).

Here we use retrograde and anterograde tracing to identify definitively which regions of the cerebral cortex project to the IC, and used immunohistochemistry to define the neuronal targets of these connections. We demonstrate that extensive projections from sensory, motor, and executive cortices innervate all subdivisions of the IC. These findings show that our understanding of perceptual processing, and specifically the contribution of the auditory midbrain, must take account of these several and diverse sources of top-down cortical input. We speculate that such feedback mechanisms may provide multisystem prediction and feedback signals that are essential for an individual to make sense of, and respond to, the auditory world.

\section{Materials and Methods}

Animals. Experiments were performed in accordance with the terms and conditions of a Project Licence issued by the UK Home Office under the Animals (Scientific Procedures) Act 1986 and with the approval of the Local Ethical Review committee of Newcastle University. Male Listerhooded rats (Rattus norvegicus, 250-350 g, age 9-15 weeks) were obtained from Charles River and housed in the Newcastle University Comparative Biology Centre in keeping with the ARRIVE (Animal Research: Reporting of In Vivo Experiments) guidelines. Rats were acclimatized for at least $5 \mathrm{~d}$ before use and were maintained in an enriched environment under a $12 \mathrm{~h}$ light/dark cycle (lights on 7:00 A.M.to 7:00 P.M.) with access to food and water ad libitum.

Surgical procedures. Animals were heavily sedated with ketamine/medetomidine ( $\approx 15 / 0.2 \mathrm{mg} / \mathrm{kg}$, i.p.) and the scalp was shaved. Local anesthetic cream (Emla) was applied to the nose, and a pediatric nasogastric tube was inserted into one nostril for the delivery of isoflurane in $\mathrm{O}_{2}$. The concentration of isoflurane was adjusted $\left(1 \%-4 \%\right.$ in $\left.\approx 0.1 \mathrm{~L} / \mathrm{min} \mathrm{O}_{2}\right)$ to provide a surgical plane of anesthesia throughout. Animals were placed in a stereotaxic frame using atraumatic ear bars (David Kopf Instru-
Table 1. Anterograde injection sites $^{a}$

\begin{tabular}{lll}
\hline Rat identifier & Left & Right \\
\hline Anterograde 1 & - & Auditory* \\
Anterograde 2 & - & Auditory $^{*}$ \\
Anterograde 3 & - & Auditory \\
Anterograde 4 & - & Auditory \\
Anterograde 5 & Visual & Prefrontal \\
Anterograde 6 & - & Motor $^{*}$ \\
Anterograde 7 & Prefrontal & Visual* $^{*}$ \\
Anterograde 8 & Visual* & Prefrontal \\
Anterograde 9 & Prefrontal & Somatosensory \\
Anterograde 10 & Somatosensory* & Visual \\
\hline
\end{tabular}

${ }^{a}$ TRITC-labeled dextran (red) was always injected into the right hemisphere, and fluorescein-labeled dextran (green) was injected into the left hemisphere.

*The animals/injections used in the immunohistochemistry studies.

ments) with the tooth bar set at $-0.3 \mathrm{~mm}$ (flat skull position). Body temperature was measured with a rectal probe and maintained at $37^{\circ} \mathrm{C}$ with a homeothermic blanket (Harvard Instruments). A midline incision was made in the scalp, and the periosteum was removed to reveal the skull.

For retrograde tracing experiments $(n=8)$, a craniotomy was performed over both ICs (coordinates from bregma: AP $-8.5 \mathrm{~mm}, \mathrm{ML} \pm 2.0$ $\mathrm{mm}$ ) leaving a bridge of bone in the midline. Retrobeads (Lumafluor) were injected using a Nanoject programmable injector (Drummond Scientific) fitted with a borosilicate glass capillary pipette $(1.14 \mathrm{~mm}$ OD, $0.53 \mathrm{~mm}$ ID, Drummond Scientific) pulled to a fine tip and broken back to allow filling. Pipettes were preloaded with Aloe Vera and a small volume of Retrobeads at the tip end. Red Retrobeads were injected into the right IC and green Retrobeads into the left. In each case, the pipette was initially lowered into the ICC to a depth of $-4.5 \mathrm{~mm}$ from the brain surface, and $200 \mathrm{nl}$ of Retrobeads were injected over a period of $2 \mathrm{~min}$. The pipette was left in situ for $2 \mathrm{~min}$ before being raised to $-3.3 \mathrm{~mm}$ where a further $200 \mathrm{nl}$ was injected, and then to $-2.3 \mathrm{~mm}$ where a final $200 \mathrm{nl}$ injection was made. To reduce the likelihood of the injection being drawn upward through the tissue following the final injection, the pipette was left in place for $5 \mathrm{~min}$ before it was removed.

For anterograde tracing experiments, TRITC- or fluorescein-labeled dextran was injected into either one or two cortical regions $(n=10$; Table 1). A glass capillary pipette (1.14 mm OD, $0.53 \mathrm{~mm}$ ID, Drummond Scientific) preloaded with TRITC- or fluorescein-labeled dextran (10,000 MW, D1816 and D1821, Invitrogen, $10 \mathrm{mg} / \mathrm{ml}$ in sterile $0.9 \%$ saline) was fitted to the Nanoject injector. Injections $(200 \mathrm{nl} /$ site $\times 2$ $\mathrm{min}$ ) were made at two or three positions in each region. Cortical regions targeted were as follows: AC (craniotomy at AP -5.2, ML $4.8 \mathrm{~mm}$, injector angled at $30^{\circ}-20^{\circ}$ away from the midline, injection sites corresponding to $\mathrm{AP}-5.2$, ML 7.4, DV $-5.6 \mathrm{~mm}, \mathrm{AP}-5.2$, ML 6.8, DV $-4.2 \mathrm{~mm}$, and AP - 5.2, ML 5.8, DV - 1.6 mm); PFC (AP 3.2, ML 0.5, DV -4.3, -2.8 , and $-1.6 \mathrm{~mm}$ ); motor cortex (injections: AP 3.2, ML 1.0, DV -1.0 $\mathrm{mm}$, and AP 3.2, ML $-1.7, \mathrm{DV}-2.2 \mathrm{~mm}$ ); somatosensory cortex (AP 2.1, ML 5.0, DV -1.9 and $-1.0 \mathrm{~mm}$ ); and visual cortex (VC; AP -8.0, ML 2.5, DV $-0.8 \mathrm{~mm}$, and AP - 8.0, ML 4.1, DV $1.30 \mathrm{~mm}$, and AP - 8.0, ML 5.1, DV $-1.0 \mathrm{~mm}$ ). TRITC-labeled dextran (red) was always injected into the right hemisphere, and fluorescein-labeled dextran (green) was injected into the left hemisphere. Following injection, the pipette was left in place for $5 \mathrm{~min}$ to minimize the spread of the tracer.

Toward the end of the surgical procedure, animals received meloxicam (15 $\mu$ g, s.c.) to provide analgesia during recovery. The scalp wound was then stitched using Vicryl 4.0 suture (Ethicon) and local anesthetic cream (Emla) was applied to the wound. Animals were allowed to recover from the anesthetic in a warm environment and were then returned to their home cage and normal housing conditions. The next morning, a second dose of meloxicam (15 $\mu$ g, s.c.) was administered to provide ongoing analgesia.

Tissue collection and processing. Between 48 and $60 \mathrm{~h}$ following Retrobead injection, or 7-8 d following injection of dextran, animals were deeply anesthetized with pentobarbital $(\approx 400 \mathrm{mg} / \mathrm{kg})$ and transcardially perfused with $\approx 100 \mathrm{ml}$ heparinized $0.1 \mathrm{M}$ PBS composed of the following 
a

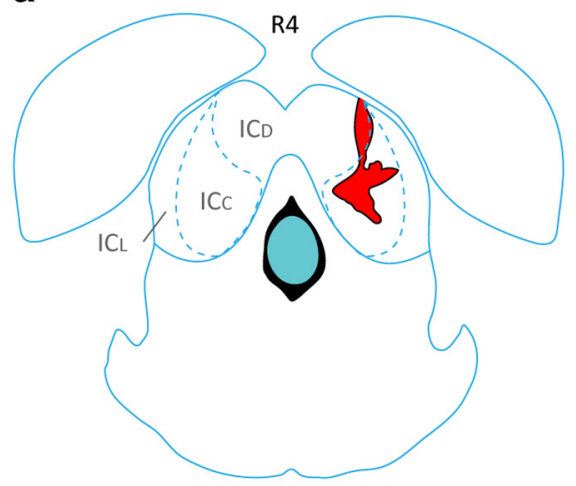

Bregma $-8.80 \mathrm{~mm}$ b

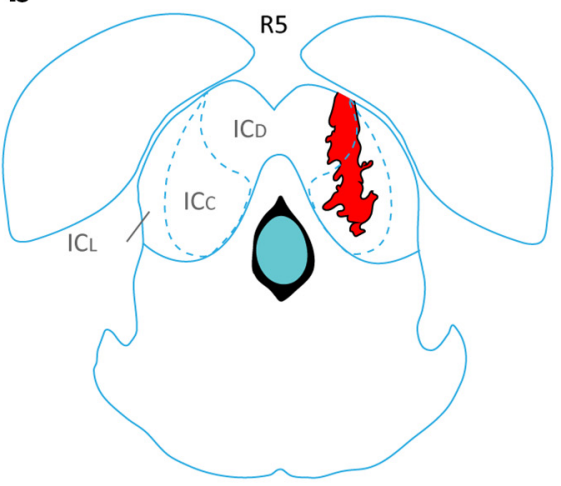

C

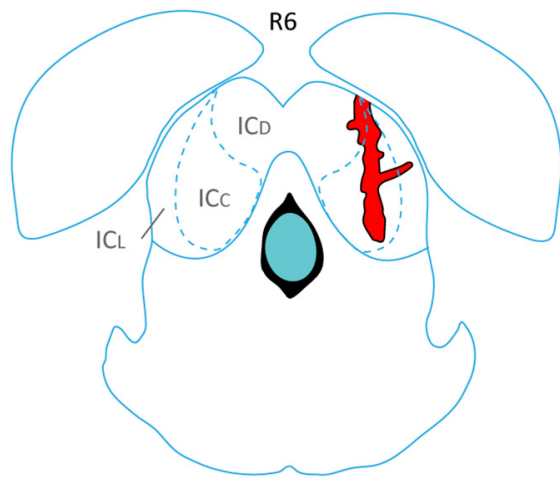

Figure 1. Sites of Retrobead injection in the right IC. For the 3 animals used for quantitative analysis ( $\boldsymbol{a}, \mathrm{R} 4 ; \boldsymbol{b}, \mathrm{R} 5$; and $\boldsymbol{c}$, R6), mosaic images were taken and the outline of each injection site was traced in Adobe Illustrator and superimposed on corresponding atlas outlines redrawn from Paxinos and Watson (1998). Figures show the rostrocaudal midpoint of the injection.

$(\mathrm{mm}): 66 \mathrm{NaCl}, 16 \mathrm{Na}_{2} \mathrm{HPO}_{4}, 3.8 \mathrm{KH}_{2} \mathrm{PO}_{4}$, followed by $\approx 100 \mathrm{ml} 4 \% \mathrm{PFA}$ in PBS. The brain was removed, postfixed overnight in $4 \% \mathrm{PFA}$, and then cryoprotected in $30 \%$ sucrose in PBS. Cryoprotected brains were stored at $-80^{\circ} \mathrm{C}$ until sectioning.

For retrograde tracing experiments, the brain was sectioned on a cryostat from the cerebellum to the olfactory bulb. Coronal sections (30 or $40 \mu \mathrm{m})$ were collected onto gelatin subbed slides, air dried, and stored at $-20^{\circ} \mathrm{C}$. Before imaging, slides were dipped in DAPI ( $\left.1 \mu \mathrm{g} / \mathrm{ml}, 5 \mathrm{~min}\right)$, air dried, and coverslipped with Fluoroshield mounting medium (SigmaAldrich).

For anterograde tracing experiments, the cortical injection sites and the ICs were sectioned. Coronal sections $(30 \mu \mathrm{m})$ were cut on a rotary microtome and collected into antifreeze solution (30\% ethylene glycol, $30 \%$ sucrose, $1 \%$ polyvinyl pyrrolidone- 40 in PBS) (Watson et al., 1986) and stored at $-20^{\circ} \mathrm{C}$. Sections from the injection sites and the ICs were mounted on glass slides, dipped in DAPI $(1 \mu \mathrm{g} / \mathrm{ml}, 5$ $\min )$, rinsed and coverslipped with Fluoroshield mounting medium (Sigma-Aldrich).

One mid rostrocaudal IC section from each animal was immunolabeled for GABA (rabbit anti-GABA antibody 1:1000, Sigma-Aldrich, catalog \#A2052, RRID:AB_477652), and the neuronal marker NeuN (mouse anti-NeuN antibody 1:1000, Merck Millipore, catalog \#MAB377, RRID:AB_2298772). A second section was immunolabeled for nitric oxide synthase (nNOS; rabbit anti-nNOS, 1:3000, Sigma-Aldrich, catalog \#N7280, RRID:AB_260796) to identify the subdivisions of the IC (see below). Sections were washed with PBS $(3 \times 10 \mathrm{~min})$, incubated in $1 \%$ $\mathrm{NaBH}_{4}$ for antigen retrieval (30 min), and washed in PBS $(3 \times 10 \mathrm{~min})$ before being incubated ( $4 \mathrm{~h}$ at room temperature followed by overnight at $8^{\circ} \mathrm{C}$ ) in a mixture containing the primary antibodies made up in a block buffer comprising 1\% BSA (Sigma-Aldrich), $0.1 \%$ porcine gelatin $(\mathrm{BDH})$, and $50 \mathrm{~mm}$ glycine (Thermo Fisher Scientific) in PBS. Sections were washed again $(3 \times 10 \mathrm{~min}$ PBS $)$ before incubation $(2 \mathrm{~h}$ at room temperature) with a mixture of AlexaFluor- 488 or -568 goat anti-rabbit secondary (1:500, Invitrogen) and biotinylated horse anti-mouse secondary (1:500, Vector Labs) in 5\% normal goat serum (Sigma-Aldrich) in PBS. Sections were washed in PBS $(3 \times 10 \mathrm{~min})$ and incubated with Cy5 streptavidin (1:500, Invitrogen) in PBS ( $1 \mathrm{~h}$ at room temperature). Finally, sections were washed in PBS (10 min), incubated in DAPI (1 $\mu \mathrm{g} / \mathrm{ml}, 10 \mathrm{~min}$ ), washed again in PBS (10 min), and dipped in distilled water before mounting on plain glass slides. Slides were coverslipped with Fluoroshield (Sigma-Aldrich).

Imaging. For retrograde tracing experiments, the injection sites were first examined to verify proper placement of the injection and to determine the extent of the local spread of the tracer. Only labeling from appropriately placed injections ( 15 of 16) was further examined. Next, every third slide was inspected for the presence of red and green fluorescent Retrobeads on a widefield microscope (Nikon NiE equipped with an
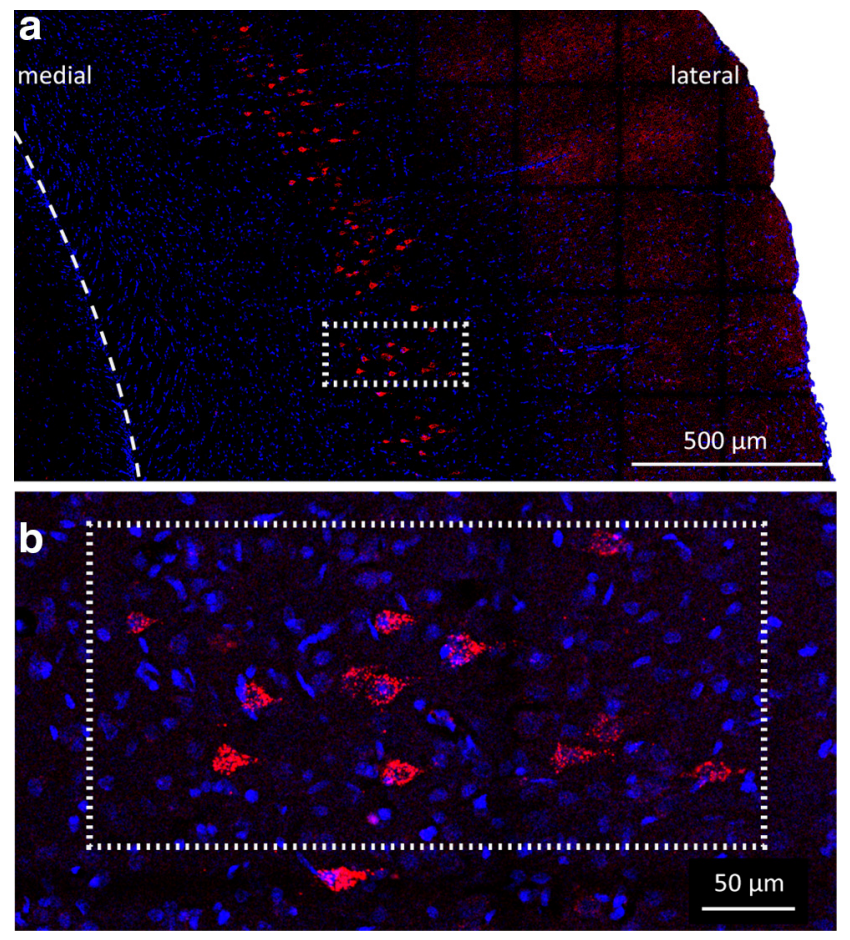

Figure 2. Neurons in the ipsilateral $\mathrm{AC}$ retrogradely labeled from the IC. Following injection of red Retrobeads into the right $I C / / C D$, Retrobeads are found in neurons in the right $A C$. Nuclei are labeled with DAPI (blue). $\boldsymbol{a}$, Retrogradely labeled cells are concentrated in a narrow band corresponding to layer $\mathrm{V}$ of AC. $\boldsymbol{b}$, The distribution of beads in the somata highlights the pyramidal cell morphology of the retrogradely labeled neurons.

Andor Zyla 5.2. camera). Detailed notes of areas with retrogradely labeled cells were made on a copy of the Brain Atlas in Stereotaxic Coordinates (Paxinos and Watson, 1998).

Three animals in which the injection of Retrobeads was of very similar size and centered within the ICC at a mid rostrocaudal level were chosen for quantitative analysis. For these 3 animals, low-power confocal mosaic images of cortical regions and the IC injection sites were acquired on a AxioObserver Z1 (Carl Zeiss), fitted with an LSM800 confocal scan head and a motorized stage using a $20 \times$ air objective $(0.8$ numerical aperture NA, $0.52 \mu$ s pixel dwell time, $X-Y$ dimension $1024 \times 1024$, bit depth 8 with linear look-up tables [LUTs]). The images were examined, and cells containing fluorescent beads were manually tagged in FIJI/ImageJ (Schindelin et al., 2012) 

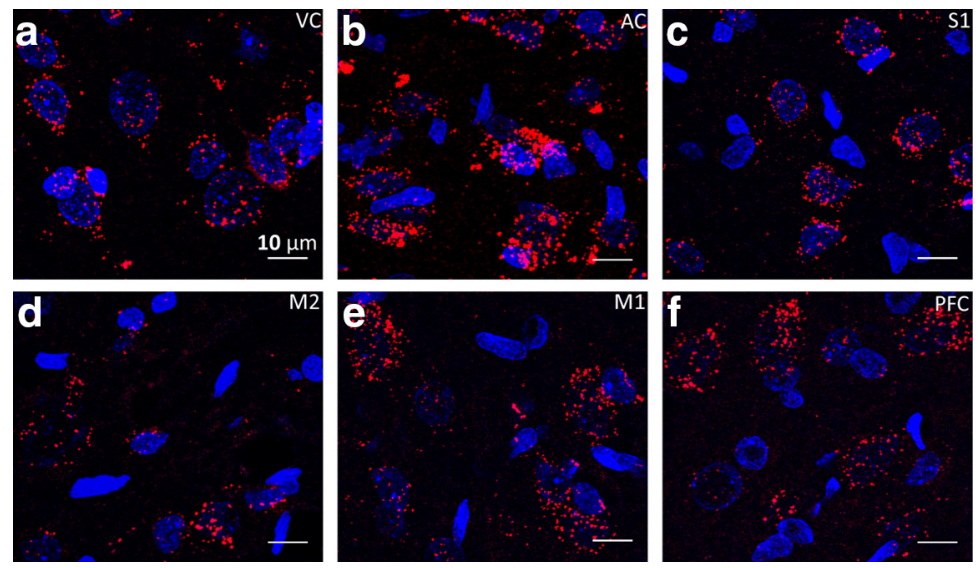

Figure 3. Multiple cortical regions are retrogradely labeled from the IC. High-power maximum intensity projections from confocal $Z$ stacks showing red Retrobeads in neurons in cortical regions ipsilateral to the injection site. $\boldsymbol{a}$, VC. $\boldsymbol{b}, A C$. c, Primary somatosensory cortex (S1). $\boldsymbol{d}$, Secondary motor cortex (M2). e, Primary motor cortex (M1).f, PFC. Nuclei are stained with DAPI (blue).

Table 2. Retrogradely labeled cells in cortical regions ${ }^{a}$

\begin{tabular}{|c|c|c|c|c|c|c|c|c|c|}
\hline \multirow{2}{*}{$\begin{array}{l}\text { Area } \\
\left(\mathrm{mm}^{2}\right)\end{array}$} & & \multicolumn{4}{|c|}{ Ipsilateral } & \multicolumn{4}{|c|}{ Contralateral } \\
\hline & & R 4 & R 5 & R6 & Mean & R 4 & R5 & R 6 & Mean \\
\hline PFC & Count & 439 & 595 & 504 & 513 & 362 & 510 & 415 & 429 \\
\hline (10.57) & $\%$ & 54.9 & 54.7 & 56.2 & 55.3 & 45.1 & 45.3 & 43.8 & 44.7 \\
\hline M2 & Count & 315 & 430 & 262 & 336 & 225 & 294 & 190 & 236 \\
\hline (6.31) & $\%$ & 57.7 & 60.5 & 59.1 & 59.1 & 42.3 & 39.5 & 40.9 & 40.9 \\
\hline M1 & Count & 491 & 565 & 638 & 565 & 401 & 480 & 549 & 477 \\
\hline (11.91) & $\%$ & 56.6 & 56.4 & 55.0 & 56.0 & 43.4 & 43.6 & 45.0 & 44.0 \\
\hline S2 & Count & 73 & 104 & 120 & 99 & 56 & 80 & 100 & 79 \\
\hline (0.98) & $\%$ & 62.2 & 58.2 & 57.2 & 59.2 & 37.8 & 41.8 & 42.8 & 40.8 \\
\hline S1 & Count & 518 & 493 & 430 & 480 & 403 & 355 & 345 & 368 \\
\hline$(13.60)$ & $\%$ & 57.3 & 60.2 & 57.4 & 58.3 & 42.7 & 39.8 & 42.6 & 41.7 \\
\hline AuV & Count & 145 & 153 & 59 & 119 & 46 & 51 & 20 & 39 \\
\hline (1.41) & $\%$ & 75.7 & 75.1 & 75.2 & 75.3 & 24.3 & 24.9 & 24.8 & 24.7 \\
\hline $\mathrm{AuD}$ & Count & 208 & 169 & 57 & 145 & 47 & 60 & 20 & 42 \\
\hline (1.70) & $\%$ & 81.5 & 74.4 & 74.0 & 76.6 & 18.5 & 25.6 & 26.0 & 23.4 \\
\hline Au1 & Count & 170 & 171 & 124 & 155 & 52 & 55 & 40 & 49 \\
\hline (2.45) & $\%$ & 76.8 & 75.9 & 75.6 & 76.1 & 23.2 & 24.1 & 24.4 & 23.9 \\
\hline V2 & Count & 164 & 103 & 64 & 110 & 83 & 55 & 24 & 54 \\
\hline (9.15) & $\%$ & 68.3 & 69.5 & 73.7 & 70.5 & 31.7 & 30.5 & 26.3 & 29.5 \\
\hline V1 & Count & 31 & 42 & 68 & 47 & 7 & 19 & 22 & 16 \\
\hline (8.34) & $\%$ & 81.6 & 69.4 & 75.9 & 75.6 & 18.4 & 30.6 & 24.1 & 24.4 \\
\hline
\end{tabular}

${ }^{a}$ Numbers of Retrobead-labeled cells in each cortical region in each of three representative animals (R4, R5, and R6). Percentages refer to the total number of labeled cells in the ipsilateral and contralateral hemispheres for each area for each individual animal. Cells were counted in the representative sections shown in Figures 4 and 5 in each animal. However, regions differ in size, and some appear in more than one section; thus, the total area of each cortical region counted (shown in parentheses) varies between regions. Comparisons of cell counts cannot be used to infer the degree of connectivity different cortical areas have with the IC. M2, Secondary motor cortex; M1, primary motor cortex; $\mathrm{S} 2$, secondary somatosensory cortex; $\mathrm{S1}$, primary somatosensory cortex; AuV, secondary auditory cortex ventral zone; Aud, secondary auditory cortex dorsal zone; Au1, primary auditory cortex; V2, secondary visual cortex; V1, primary visual cortex.

using the Cell Counter plugin. Since the red beads were more easily distinguished than the green beads, for quantification purposes, we counted only cells containing red beads in the cortices both ipsilateral and contralateral to the injection site.

For anterograde tracing experiments, low-power confocal mosaic images $(\times 20$ air, $0.8 \mathrm{NA}, 1024$ pixels in the $X-Y$ dimension, $0.52 \mu$ s pixel dwell time, bit depth 8 with linear LUTs) of the injection sites, were first acquired from DAPI-labeled sections to verify the proper placement of injections. Next, confocal mosaic images $(\times 40$ oil, $1.3 \mathrm{NA}, 1024$ pixels in the $X-Y$ dimension, $6.06 \mu$ s pixel dwell time, bit depth 16 with linear LUTs) of the whole IC at a mid rostrocaudal level were acquired in a section immunolabeled for nNOS, and the presence of red and green dextran in the three subdivisions of the IC (ICC, ICD, and ICL) was examined. The borders of these subdivisions were delineated by the dis- tribution of labeling for nNOS. ICD and ICL are characterized by neurons with cytoplasmic labeling for nNOS, whereas the ICc contains few neurons that label cytoplasmically (Coote and Rees, 2008; Olthof et al., 2019). This difference makes the borders between the subdivisions easy to distinguish (see Fig. 7a). Since TRITCdextran was more easily visualized and quantified than fluorescein-dextran, in 6 cases, we examined only the distribution of red dextran in both hemispheres after injection into the right hemisphere (see Fig. $6 a-d$ ). In 2 cases (one VC injection and one somatosensory cortex injection; see Fig. $6 b, d$ ), we examined the distribution of fluorescein-dextran after injection into the left hemisphere.

To allow a qualitative examination of the relative densities of innervation of the three IC subdivisions (see Fig. $7 b-f$ ), fluorescent puncta of dextran were captured using the "analyze particles" function in ImageJ and a "mask" was made for each particle. As the puncta are too small to be visible at low power, for illustration purposes, we expanded each mask digitally by a radius of 15 pixels using Adobe Photoshop.

To determine whether dextran labeling in terminals was associated with GABAergic or non-GABAergic (putative glutamatergic) neurons, confocal $Z$ stacks of varying depths $(7-15 \mu \mathrm{m})(\times 63$ oil, $1.40 \mathrm{NA}, 1.03 \mu \mathrm{s}$ pixel dwell time, $X$ - $Y$ dimensions $1024 \times 1024, Z$ step $0.26 \mu \mathrm{m}$, bit depth 8 with linear LUTs) were taken from the immunolabeled sections. Representative $Z$ stacks were taken in the ICD ( 2 stacks per animal), ICC (3 stacks per animal), and ICL ( 1 stack per animal). $Z$ stacks were cropped post hoc to $7 \mu \mathrm{m}$ thickness. The presence of red dextran was imaged in sections in which GABA had been labeled with AlexaFluor-488 (green), and green dextran was imaged in sections in which GABA had been labeled with AlexaFluor-568 (red).

Using Imaris (Bitplane), the DAPI signal was rendered to a "surface" and the dextran labeling was rendered into "spots." The proximity of the dextran "spots" to DAPI "surface" was measured, allowing us to assess whether dextran-labeled terminals were in close proximity $(\leq 3 \mu \mathrm{m})$ to cell somata.

We immunolabeled for NeuN to visualize more of the cell body and proximal processes of the IC neurons to allow us to examine whether dextran-labeled terminals contacted somatic or dendritic sites. However, we noted that many DAPI-labeled nuclei in the IC that were in close proximity to dextran-labeled terminals (in some cases also labeled for GABA) and thus presumed to be neurons, did not label for NeuN. Hence, we used the DAPI nuclear signal rather than NeuN to define cell somata.

Experimental design and statistical analysis. This study describes largely qualitative data on the distribution of retrograde and anterograde tracers in the brain.

For retrograde tracing experiments, 8 animals received injections of Retrobeads: red in the right and green in the left IC. For each animal, sections through the whole brain were visually inspected using a widefield microscope. The presence of both red and green beads in both hemispheres was recorded. For quantification, we selected six sections (distributed rostrocaudally through the brain) from each of 3 animals. All cells containing red Retrobeads were counted in each section in both hemispheres, dividing cortical areas according to the atlas of Paxinos and Watson (1998). Descriptive statistics (mean \pm SEM) for the raw cell counts and for the percentage in each hemisphere are presented. No statistical comparisons were made.

For anterograde tracing experiments, 10 animals received injections of fluorescent dextran: 5 received one injection (right side only) and 5 received two injections (one right, one left). Red and green dextran was injected into the right and left hemispheres, respectively (Table 1). From these animals, the whole of the IC was sectioned and visually inspected using a widefield microscope and the distribution of dextran recorded. Distribution of the terminal fields in the subdivisions of the IC were 
studied using one IC section from 1 animal with an injection in each of the cortical ROIs. To examine lateralization, one IC section from 1 animal with an injection in the motor cortex and 1 animal with an injection in the AC were selected. Dextran spots were counted in five $Z$ stacks of ROIs $\left(160 \mu \mathrm{m}^{2}, 7 \mu \mathrm{m}\right.$ thickness: one in ICD and ICL, and 3 in ICC) in both hemispheres. Descriptive statistics (mean and SEM) of the percentage of dextran spots in each hemisphere are presented. No statistical comparisons were made. To examine the distribution of dextran around GABA-labeled elements, one section from individual animals with injections in the prefrontal $(n=1)$, motor $(n=1)$, auditory $(n=2)$, visual $(n=2)$, and somatosensory $(n=2)$ cortices were immunolabeled for GABA. These sections were visually inspected at high power $(\times 40)$, and photographs representative of the distribution of dextran relative to GABA-labeled elements were taken.

\section{Results}

\section{Retrograde labeling}

Injection sites

We injected red Retrobeads into the right IC and green Retrobeads into the left IC of 8 rats (see Materials and Methods). Retrobead injection sites in all animals were inspected using a widefield microscope which revealed the presence of many bright beads. In all but 1 case, Retrobead injections were well placed: one green Retrobead injection was misplaced, and the data from this injection were discounted. Correctly placed injections $(n=15)$ were centered in the ICC and included the lateral part of the ICD: in some cases, there was some spread of Retrobeads into ICL. All injections were contained entirely within the boundaries of the IC. Notably, there was no upward spread of Retrobeads into the visual cortex (VC) that overlies the IC. From the larger group of injections, the red Retrobead injections in 3 animals (R4, R5, and R6) were chosen for quantitative analysis. Figure 1 shows the extent of the red Retrobead injection in in the right IC in these animals traced from $\times 20$ confocal mosaic images.

\section{Retrograde labeling from the IC is seen in multiple cortical areas} in both the ipsilateral and contralateral hemispheres

As a positive control for our retrograde labeling, we first examined the presence of Retrobeads in the AC ipsilateral and contralateral to the injection site (henceforth referred to as the ipsilateral and contralateral cortices). As anticipated, Retrobeads injected in the ICC/ICD labeled many neurons in both the ipsilateral and contralateral AC, predominantly at a depth consistent with layer $\mathrm{V}$ (Fig. 2a). Labeling was evident as distinct bright beads, which are associated with DAPI-stained nuclei, suggesting that the beads are located within cell somata. The subcellular distribution of the Retrobeads and the orientation of the labeled cells indicate that they are pyramidal neurons (Fig. $2 b$ ).

In addition to the labeling seen in the AC, large numbers of Retrobead-labeled neurons were also seen in many nonauditory cortical areas. Indeed, labeling extended from the visual areas caudally all the way to the prefrontal regions rostrally. Large numbers of neurons containing Retrobeads were found in VC (Fig. $3 a$ ), somatosensory cortex (Fig. 3c), motor cortex (Fig. 3d,e), and prefrontal cortex (PFC) (Fig. 3f; Table 2). As in AC (Fig. 3b), Retrobeads were predominantly in the cell soma; however, compared with the labeling in $\mathrm{AC}$ (Fig. $3 b$ ) in nonauditory cortical areas (Fig. $3 a, c-f$ ), the density of Retrobeads in each cell appeared lower.

\section{Visual cortex}

Retrogradely labeled neurons were found in both ipsilateral and contralateral visual cortices (Figs. $4 a, b, 5 a, b$; Table 2). They were numerous in both primary VC (monocular and binocular) and secondary VC (lateral area and mediolateral area) (Figs. 4a,b, $5 a, b)$. Caudally, labeled cells were found in a wide band (Figs. $4 a$,
$5 a)$, whereas more rostrally, retrogradely labeled cells were concentrated in the deeper part of the cortical thickness (Figs. $4 b, 5 b$ ). Quantification of the labeled neurons (in two sections from each of 3 animals) revealed a consistently high degree of lateralization. Thus, although retrograde labeling was bilateral, there were far fewer labeled neurons in the contralateral compared with the ipsilateral visual cortices (Figs. $4 a, b, h, 5 a, b$; Table 2).

\section{Auditory cortex}

As expected (see Introduction), retrograde labeling was seen in both the ipsilateral and contralateral auditory cortices (Figs. $4 b, c$, $5 b, c$; Table 2). Many labeled neurons were present in the primary $\mathrm{AC}$ and both dorsal (secondary auditory cortex dorsal zone) and ventral secondary auditory cortices (Figs. $4 b, c, 5 b, c)$. On the ipsilateral side, labeled neurons were densely packed in presumed layer V with a few in layer VI; whereas on the contralateral side, the labeled neurons were scattered over a wider band (Figs. $4 b, c$, $5 b, c)$. As in the VC, we observed far fewer retrogradely labeled neurons in the contralateral compared with the ipsilateral AC (Fig. 4h; Table 2).

\section{Somatosensory cortex}

A second major nonauditory sensory cortical region that contained a substantial number of retrogradely labeled neurons, was the somatosensory cortex. As was the case in the auditory and visual cortices, in the somatosensory cortex, labeling was present in both ipsilateral and contralateral hemispheres (Figs. $4 c-e$, $5 c-e$; Table 2). Primary somatosensory regions with large numbers of retrogradely labeled cells included the trunk, forelimb, hindlimb, dorsal zone, and jaw areas. Although there are relatively few cells in the barrel field of Case R5 (shown in Fig. 4c), Cases R4 and R6 did have some labeled neurons in this region (Fig. $5 c$ ). Secondary somatosensory areas also contained large numbers of retrogradely labeled cells (Figs. $4 c, 5 c$ ). In more rostral sections, the width of the band of labeled cells increases consistent with the increased thickness of the cortical layers (Figs. $4 d, e, 5 d, e)$. Interestingly, while there were more labeled neurons ipsilateral than contralateral to the injected IC, this difference was not as great as that observed in the auditory and visual cortices (Fig. 4h; Table 2).

\section{Motor cortex}

Outside the sensory domain, we also found retrograde labeling from the IC in the motor cortex. Here, as in other cortical regions, labeling was evident in both ipsilateral and contralateral sides. Many labeled neurons were seen in both the primary motor cortex and secondary motor cortex (Figs. $4 d-f, 5 d-f$; Table 2 ), and labeling extended along the whole rostrocaudal extent of the motor cortices. On both ipsilateral and contralateral sides, labeled neurons were dispersed more widely through the cortical thickness. It was notable that, in motor areas, the lateralization pattern of retrograde labeling resembled that seen in the somatosensory regions in that only marginally fewer labeled neurons were found in the contralateral compared with the ipsilateral side (Fig. 4h; Table 2).

\section{Prefrontal cortex}

The PFC lies at the most rostral end of the cerebral cortex and comprises both the medial prefrontal and orbitofrontal regions. Following Retrobead injections into the IC, many labeled neu- 


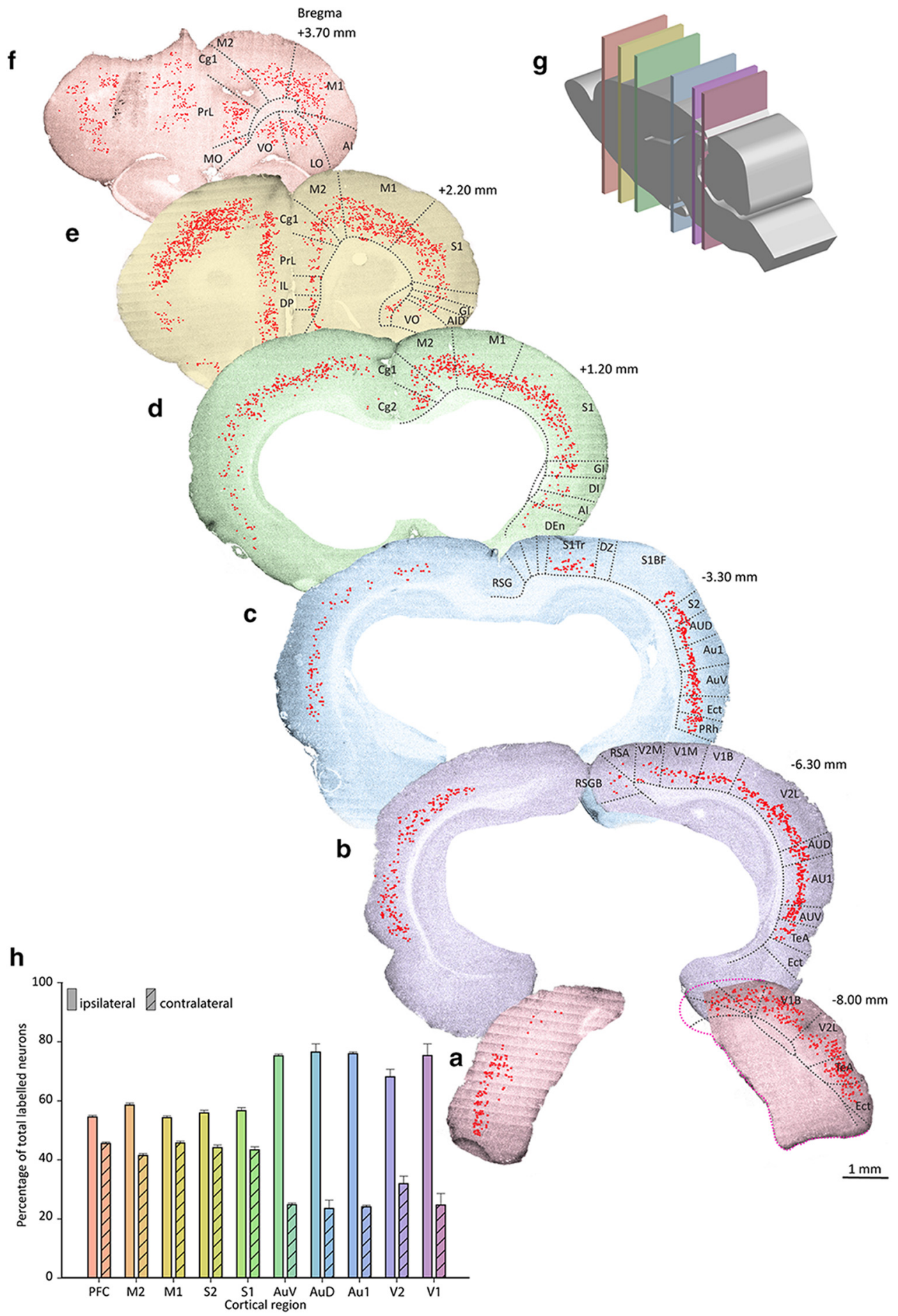

Figure 4. Retrograde cortical labeling demonstrates bilateral inputs from multiple cortical regions to IC. $\boldsymbol{a}-\boldsymbol{f}$, Confocal mosaic images of coronal cortical sections at multiple locations (shown as distance from bregma) along the rostrocaudal axis ( $\boldsymbol{g}$ ) following the injection of red Retrobeads in the right ICc/ICD in 1 animal, R5. Red markers represent cells containing Retrobeads. Cortical divisions are redrawn from the atlas of Paxinos and Watson (1998), and region names are abbreviated on the sections. $\boldsymbol{h}$, Proportions of retrogradely labeled cells in the cortex ipsilateral and contralateral to the injected IC as a percentage of total labeled neurons in each cortical region. Error bars indicate mean \pm SEM ( $n=3$ animals) derived from counts made in 2 or 3 sections per region per animal. Al, agranular insular cortex; Au1, primary auditory cortex; AuD, secondary auditory cortex dorsal zone; AuV, secondary auditory cortex, ventral zone; $\mathbf{C g 1}$, cingulate cortex area 1; Cg2, cingulate cortex area 2; Den, dorsal endopiriform nucleus; DI, dysgranular insular cortex; DP, dorsal peduncular cortex; DZ, primary somatosensory cortex dorsal zone; Ect, ectorhinal cortex; GI, granular insular cortex; IL, infralimbic cortex; LO, lateral orbital cortex; M1, primary motor cortex; M2, secondary motor cortex; M0, medial orbital cortex; Prh, perirhinal cortex; PrL, prelimbic cortex; RSA, retrosplenial agranular cortex; RSG, retrosplenial granular cortex; RSGb, retrosplenial granular b cortex; S1, primary somatosensory cortex; S1BF, primary somatosensory barrel cortex; S1Tr, primary somatosensory cortex trunk region; $\mathbf{S 2}$, secondary somatosensory cortex; TeA, temporal association cortex; V1B, primary visual cortex, binocular area; V1M, primary visual cortex, monocular area; V2L, secondary visual cortex, lateral area; V2M, secondary visual cortex, mediolateral area; V0, ventral orbital cortex. 
R4

f

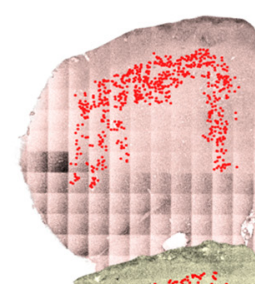

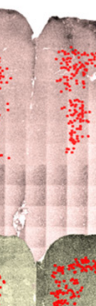

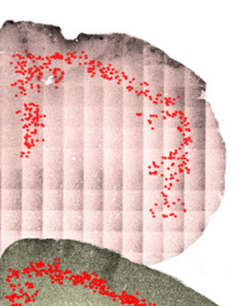

$+3.70 \mathrm{~mm}$

e
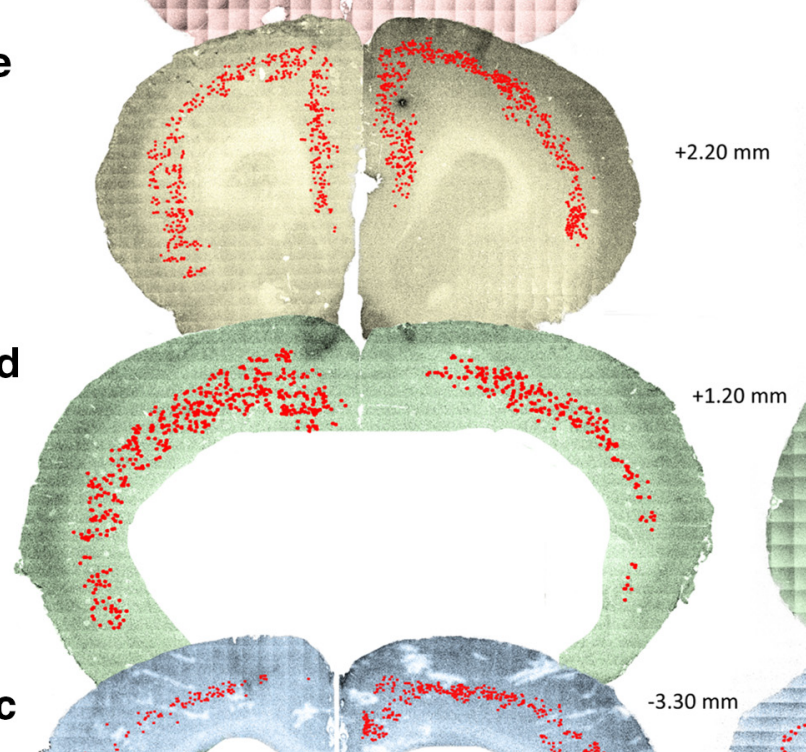

$+1.20 \mathrm{~mm}$

b
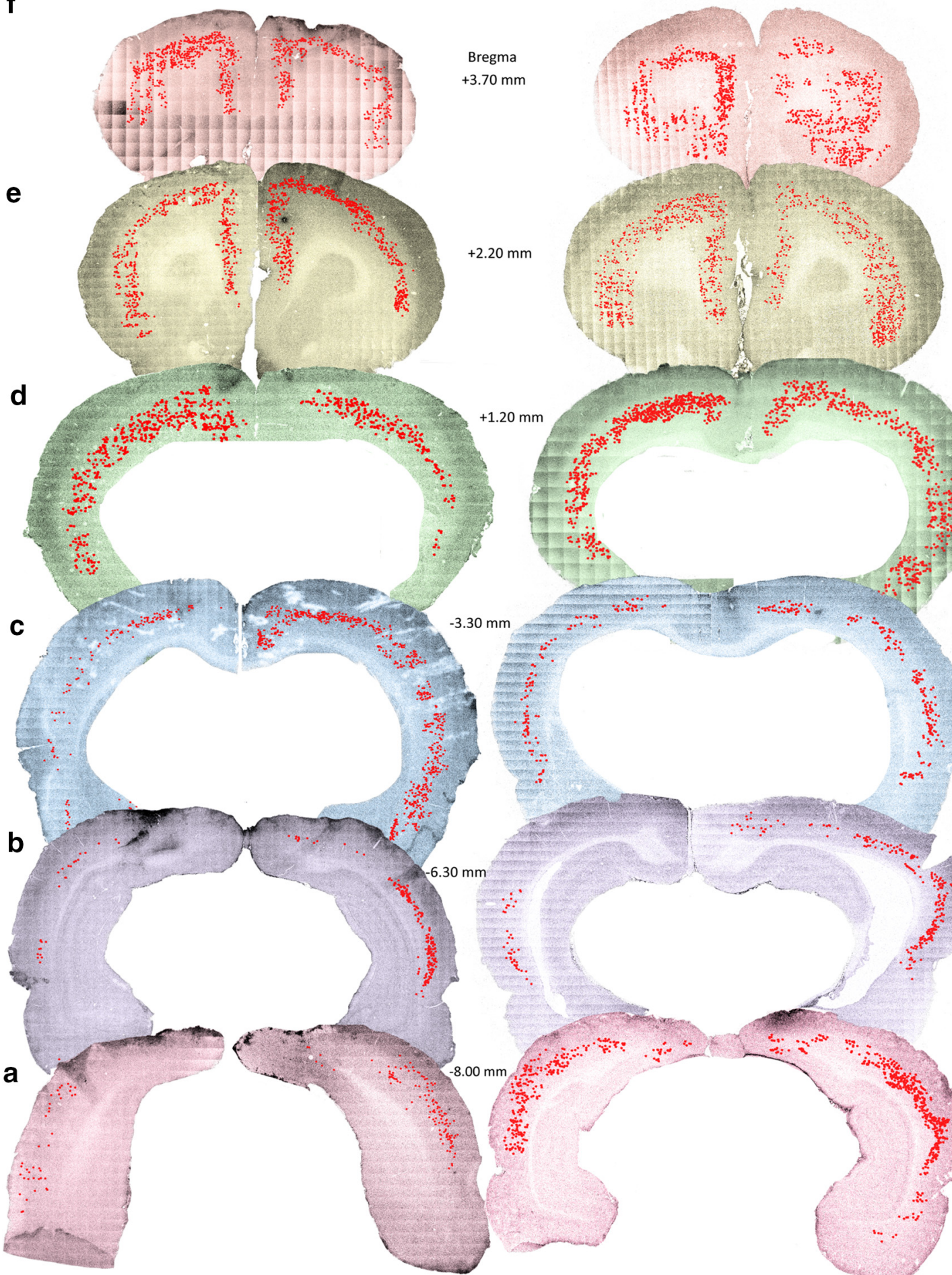

$1 \mathrm{~mm}$

$1 \mathrm{~mm}$

Figure 5. Retrograde cortical labeling demonstrating inputs from multiple cortical regions to IC in 2 further representative cases. $\boldsymbol{a}-\boldsymbol{f}$, Confocal mosaic images of coronal cortical sections at multiple locations (shown by distance from bregma) along the rostrocaudal axis (see Fig. 4g) following the injection of red Retrobeads in the right ICC/ICD in R4 (left column) and R6 (right column). Red markers represent cells containing Retrobeads. Cortical regions as marked in Figure 4a-f. These cases with case R5 (Fig. 4a-f) provide the data for the histogram in Figure $4 h$.

rons were found in the PFC of both hemispheres (Figs. $3 b, 4 d-f$, $5 d-f$; Table 2).

The medial PFC, which subserves executive function, is distinct in that its laminae lie dorsoventrally. Retrograde labeled neurons were concentrated in the cingulate (cingulate cortex area 1 and cingulate cortex area 2) and prelimbic cortices, and there were few, if any, labeled neurons in the more ventral infralimbic cortex and dorsal peduncular cortex regions. Retrogradely labeled neurons were located in the middle and deep part of the cortex thickness close to the white matter of the forceps minor. The orbitofrontal cortex, which is situated ventral to the forceps minor, integrates sensory and autonomic information. In or- 
a

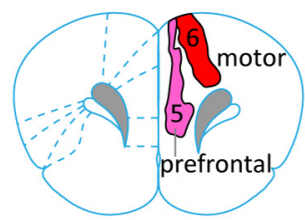

Bregma: $+3.20 \mathrm{~mm}$ b

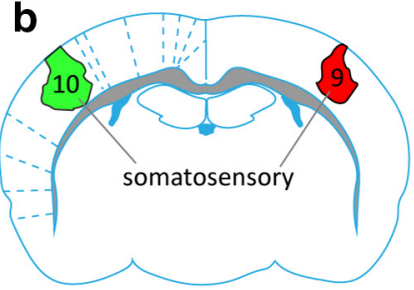

$-2.12 \mathrm{~mm}$

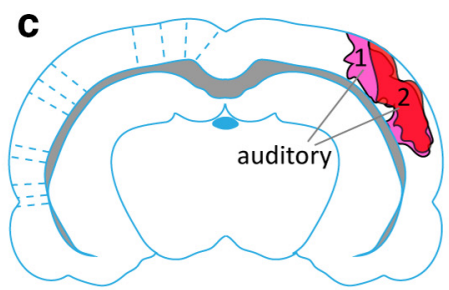

$-5.20 \mathrm{~mm}$

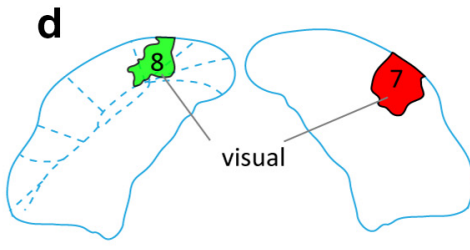

$-8.00 \mathrm{~mm}$

$1 \underline{\mathrm{mm}}$

Figure 6. Sites of anterograde tracer injection in the cortices. TRITC (red)-labeled dextran anterograde tracer was injected in the right hemisphere, and fluorescein (green)-labeled dextran was injected in the left hemisphere. For the injection sites selected for analysis, mosaic images were taken, and the outline of each injection site was traced in Adobe Illustrator and superimposed on corresponding atlas outlines redrawn from Paxinos and Watson (1998). Figures show the rostrocaudal midpoint of the injection. Each area traced is from a different animal shown on each coloured injection site. Injection sites: (a) PFC (cingulate cortex area 1 and prelimbic cortex, magenta) and motor cortex (M2, red), (b) somatosensory cortex (S1, red and green), (c) AC (primary auditory cortex; secondary auditory cortex dorsal zone; parietal association area, red and magenta), and (d) visual cortex (V1, green and V1 and V2, red).

a

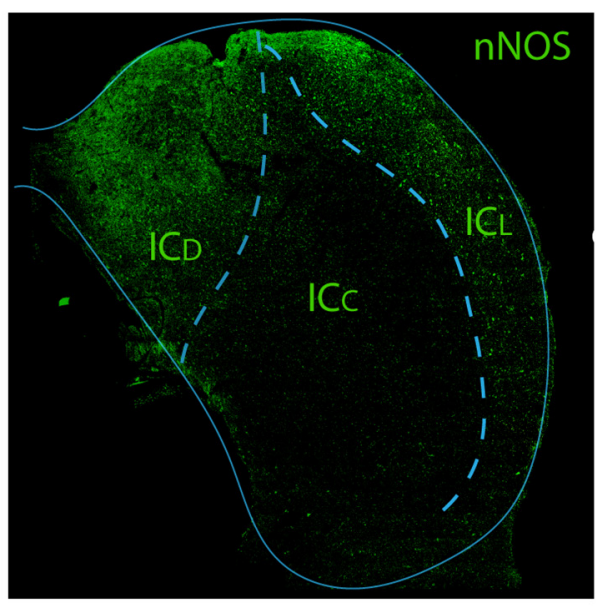

b Prefrontal cortex (A5)

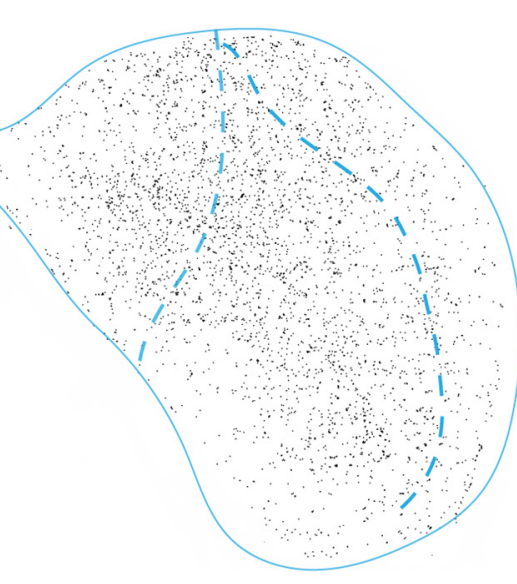

\section{Motor cortex (A6)}

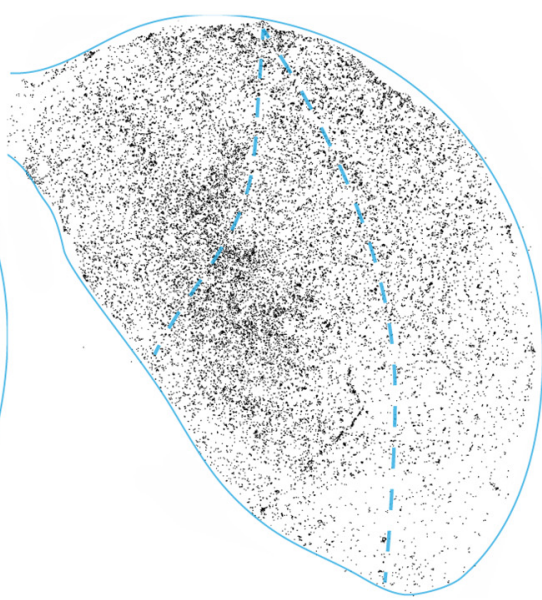

f Visual cortex (A8)

e Auditory cortex (A1)
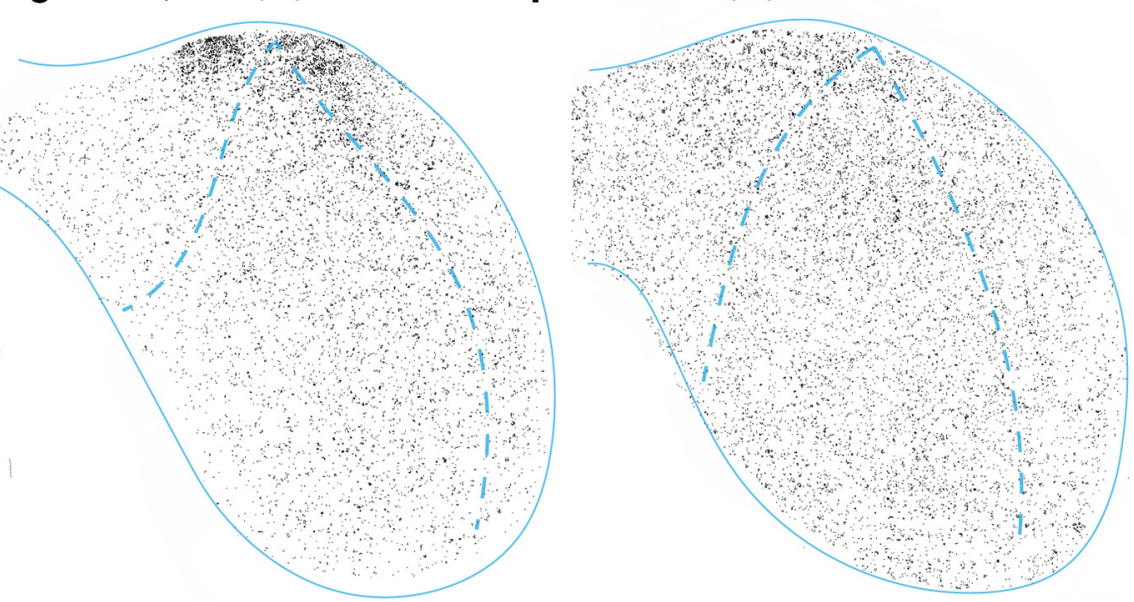

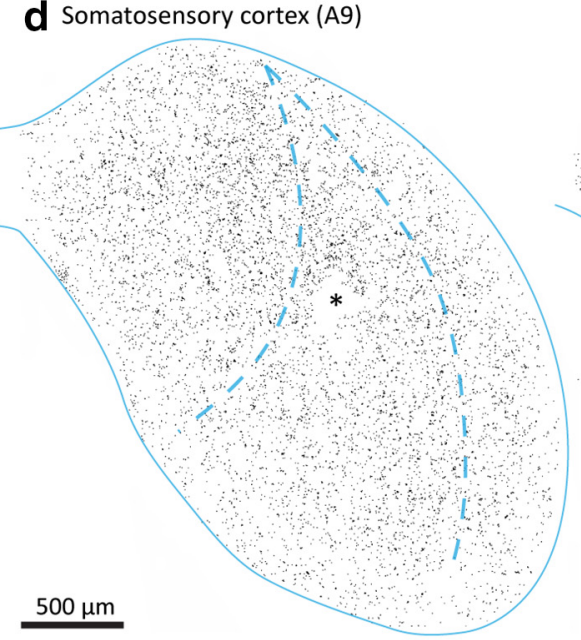

Figure 7. Distribution of dextran-labeled terminals in subdivisions of the IC following anterograde tracer injection into cortical regions. $\boldsymbol{a}$, Coronal section of IC immunolabeled for nNOS to identify the subdivisions of the IC. Cortical IC subdivisions contain neurons whose cytoplasm labels for nNOS. Anterograde labelling from the prefrontal cortex for this section is shown in (b). Similar nNOS Immunolabelling (not shown) was used to define the subdivisions in the remaining cases $(\boldsymbol{c}-\boldsymbol{f}) . \boldsymbol{b}-\boldsymbol{f}$, Coronal sections of the IC showing the distribution of dextran-labeled terminals in the IC following injection of dextran into the cortical region shown above the section. Experimental numbers correspond to Table 1. Terminals were masked and expanded to allow them to be seen at low power (see Materials and Methods). $\boldsymbol{b}, \boldsymbol{f}$, Left ICs but reflected in the vertical plane for consistency. $\boldsymbol{d},{ }^{*}$ Site of a fiducial mark where there is no tissue.

bitofrontal cortex, retrogradely labeled neurons were located more deeply and were observed in the ventral orbital cortex and lateral orbital cortex regions, with fewer labeled neurons in the medial orbitofrontal cortex. Considering the PFC as a whole (cingulate cortex area 1, cingulate cortex area 2, prelim- bic cortex, infralimbic cortex, dorsal peduncular cortex, ventral orbital cortex, medial orbital cortex, and lateral orbital cortex), the degree of lateralization was low with almost as many retrogradely labeled neurons on the contralateral side $(44 \%)$ as on the ipsilateral side (56\%) (Fig. 4h; Table 2). 
As is evident from Table 2, the number of labeled neurons counted in different cortical regions varies considerably. Such cell counts cannot, however, be used to infer differences in the degree of connectivity between cortical regions and the IC for two reasons. First, the area within a section occupied by some cortical regions is considerably greater than others (Table 2 ), and some regions extend to more than one section (Figs. $4 a-f, 5 a-f$ ). Second, we have only counted representative sections, not the whole cortex. Thus, cell counts are indicative that connections exist between specific cortical regions and the IC, but do not quantify these connections.

\section{Noncortical regions are retrogradely labeled from IC}

Following Retrobead injections into the IC, labeled cells were also observed in brainstem auditory regions known to innervate the IC (including cochlear nucleus, olivary complex, dorsal nucleus of the lateral lemniscus). In addition, we noted Retrobead-labeled cells in many subcortical forebrain regions, including olfactory bulb, caudate-putamen, hippocampus, thalamus, hypothalamus, and amygdala, as well as in midbrain and hindbrain regions, including superior colliculus and dorsal raphe. The innervation of the IC by these regions will be the subject of a second paper.

Although we concentrated our detailed analysis on the red Retrobeads and quantified retrograde labeling in only a subset of the animals, qualitatively the distribution of retrogradely labeled cells was the same for all red and green Retrobead injections.

\section{Anterograde labeling}

To verify our findings with retrograde tracing, and to examine both the regional distribution and neuronal targets of cortical inputs to the IC, we injected anterograde tracers (TRITC-labeled [red] and fluorescein-labeled [green] dextran) into cortical ROIs and examined the presence of dextran-labeled terminals in the ipsilateral and contralateral IC. For this purpose, we chose a relatively high molecular weight dextran (10,000 MW) conjugated to a fluorophore, to highlight terminals and avoid the labeling of other neuronal elements.

\section{Cortical sites of anterograde tracer injections}

At the injection site, fluorescent labeling of neuronal elements was intense and the location and dispersion of the tracer were readily visualized. All injections were correctly placed within the intended cortical ROI. Although most animals had two cortical injection sites (one red, right; one green, left), we selected just one of these sites in each animal. As a result, labeling originating from one prefrontal and one motor cortex injection, and two auditory,
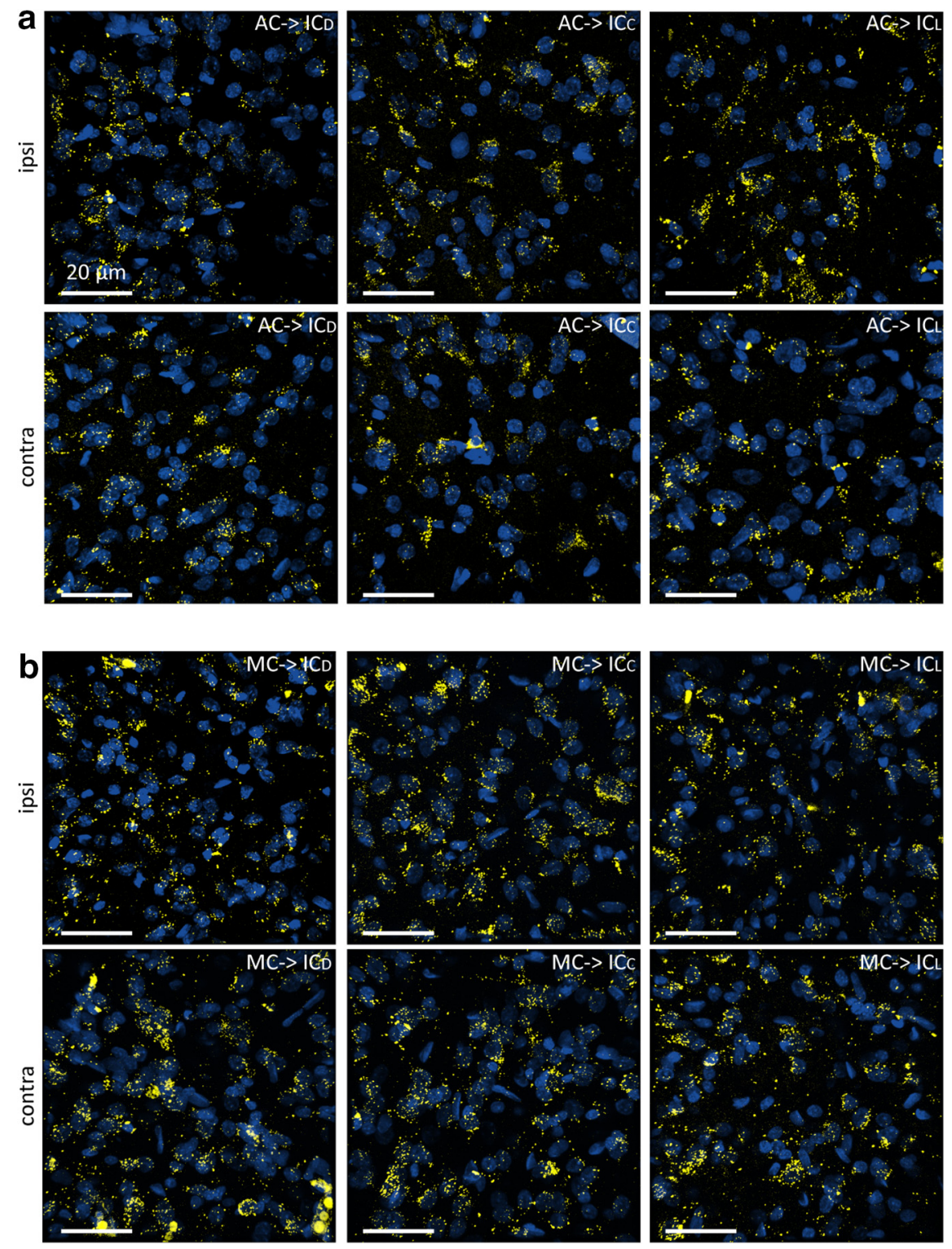

Figure 8. Anterograde labeling is present in the ICs both ipsilateral and contralateral to the cortical injection. Anterograde dextran labeling (yellow) in the three subdivisions of the IC (ICD, ICC, and ICL). Dextran injected in (a) AC (see Fig. 4c). $\boldsymbol{b}$, Motor cortex (MC, see Fig. 4a). Cell nuclei are stained with DAPI (blue).

two visual, and two somatosensory cortical injections were studied in detail (Fig. $6 a-d$ ).

\section{Anterograde labeling confirms nonauditory cortical projections} to IC

For each of the cortical ROIs highlighted by our retrograde findings (visual, auditory, somatosensory, motor, and prefrontal), dextran injection (Fig. 6; Table 1) resulted in dense anterograde labeling in the IC (Fig. 7). In all cases, dextran labeling, which was evident as individual small fluorescent puncta or clusters of puncta (presumed neuronal terminals), was present in all subdivisions (Fig. 7). Subdivisions were identified using immunolabeling for nNOS (see Materials and Methods), which distinguishes the cortical subdivisions (ICD and ICL) from the ICC (Fig. 7a). However, the different cortical sites of injection shown in Figure 6 resulted in different densities and patterns of labeling. For example, whereas the injection in the motor cortex resulted in dense labeling ventromedially in ICC and ICD, the injection in the AC resulted in dense labeling dorsally in the ICD and ICL (e.g., 

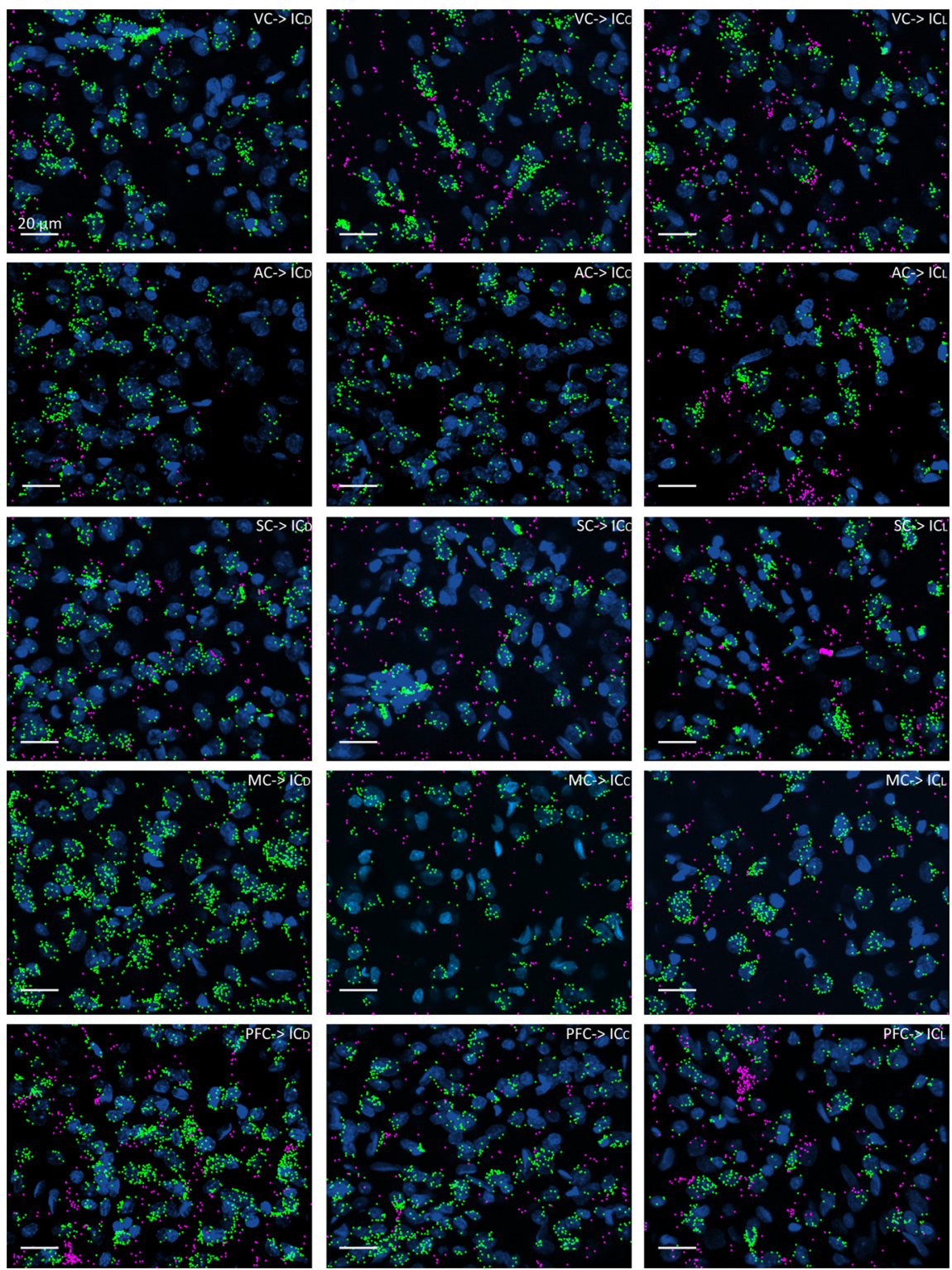

Figure 9. Anterograde tracing reveals terminals originating from multiple cortical regions target neurons in all IC subdivisions, Anterograde dextran labeling and were converted into "spots" and DAPI-stained nuclei were converted to "surfaces" (blue) using Imaris, and the proximity of spots to surfaces was determined. Green represents spots closer than $3 \mu \mathrm{m}$. Magenta represents spots further away. Distribution of anterograde-labeled terminals in ICD, ICC, and ICL (columns left to right) following injections of tracer into visual, auditory, somatosensory, motor, and prefrontal cortices (rows top to bottom).

Fig. $7 c, e$ ). However, as we only made a few tracer injections in each cortical region and these were not placed systematically, we cannot draw specific conclusions about these patterns of connectivity between cortical regions and the IC.

Our retrograde labeling showed that the largest difference in the lateralization of cortico-collicular neurons was between the AC ( $24 \%$ contralateral) and motor cortex ( $46 \%$ contralateral). Hence, for these two regions, we compared anterograde labeling in the IC ipsilateral and contralateral to the injection (Fig. 8). For both AC (Fig. 8a) and motor cortex (Fig. 8b) tracer injections, dextran-labeled terminals were observed in both ICs. Counts of dextran spots in five $Z$ stacks ( 1 in ICD, 1 in ICL, and 3 in ICC) obtained from 1 animal on both the ipsilateral and contralateral side confirmed the findings from the retrograde studies, there was evidence for highly lateralized connections from AC (62.4 \pm $2.12 \%$ ipsilateral vs $37.6 \pm 1.48 \%$ contralateral) contrasting with strongly bilateral connections from the motor cortex $(51.5 \pm 1.47 \%$ ipsilateral vs $49.5 \pm 1.97 \%$ contralateral).

To investigate the organization of labeled puncta with respect to the cellular morphology of the IC, we calculated the distance of the dextran-labeled terminals from DAPI-labeled nuclei using the Imaris (Bitplane) "spot" and "surface" functions. This analysis revealed that the majority of dextran-labeled terminals were clustered around DAPI-labeled cell nuclei, sometimes apparently extending along proximal processes but within $3 \mu \mathrm{m}$ of the nucleus (Fig. 9, green puncta). Other labeled terminals were further away from cell somata, presumably on other neuronal elements (Fig. 9, magenta puncta). This pattern of labeling was seen regardless of the cortical site of injection of the tracer or the subdivision of the IC examined.

\section{Both GABAergic and glutamatergic IC} neurones are targeted by cortical inputs Sections from our anterograde tracing studies immunolabeled for GABA revealed a high density of GABAergic neurons of various sizes and morphologies in the IC. Regardless of the cortical site at which the dextran tracer was injected, many anterogradely labeled terminals were found in close proximity both to GABA-labeled neurons and to DAPIstained nuclei of cells that did not label for GABA. Since it is well established that IC neurons contain only one of two principal neurotransmitters, glutamate or GABA (Merchán et al., 2005), these nonGABAergic cells were presumed to be glutamatergic neurons (Fig. 10d). Two populations of GABAergic neurons were discerned, large (>15 $\mu \mathrm{m}$; Fig. 10a) and small ( $<15 \mu \mathrm{m}$; Fig. 10b) (Ito et al., 2009), and dextran-labeled terminals were found in close proximity to both. In the ICL in particular, we saw GABA neurons with extensive dendrites, and dextran-labeled terminals were frequently found in close proximity to these structures (Fig. 10c). Labeled terminals were also found close to the smaller, finer, GABAergic dendrites seen in ICD and ICC (Fig. $10 c)$.

\section{Discussion}

The main finding of this study is that multiple regions of the cerebral cortex make extensive bilateral connections with all three subdivisions of the IC. In addition to previously wellestablished cortico-collicular inputs from the AC, we demonstrate substantial connections from visual, somatosensory, motor, and prefrontal regions. Retrograde tracing shows that connections from the ipsilateral side are the most abundant. However, the ipsilateral/contralateral difference is less pronounced for somatosensory, motor, and prefrontal cortices, 
where numbers of labeled neurons are nearly equal, than for the visual and auditory cortices where the difference is approximately threefold. Cortico-collicular neurons are most abundant in the deeper part of the cortical thickness, but in some regions, the motor and prefrontal cortices in particular, they are distributed more widely. Anterograde tracing suggests that these cortico-collicular inputs synapse with both GABAergic and glutamatergic neurons in all three divisions of the IC. A surprising aspect of our results is the extent to which diverse cortical projections target the ICC, the main recipient of ascending input from brainstem auditory nuclei and traditionally regarded as a center of auditory processing (Oliver, 2005; Ito and Malmierca, 2018).

\section{Technical considerations}

The combined application of retrograde and anterograde tracing methods provides convincing evidence for the existence of cortico-collicular connections from nonauditory cortical regions. Retrobeads show minimal diffusion (Katz et al., 1984) and our injections, which targeted ICC and ICD resulted in minimal spread into ICL. Importantly, no Retrobeads were observed in the overlying VC. Thus, we can be confident that the cortical labeling we observed resulted from retrograde transport from the IC. Although we quantified data using red Retrobeads from 3 exemplar animals, we saw the same pattern of labeling with both red and green Retrobeads in all 8 animals.

There were differences in the density of Retrobead labeling of neurons, both within and between regions. Retrobeads are considered efficient tracers; nevertheless, differences in the density of labeling of neighboring cortical neurons have been reported previously (Schofield et al., 2007). Such differences may relate to the number of terminals, their pattern of distribution in the IC, or even to the level of neuronal activity. Differences in labeling density are unlikely to be explained by the distance from the IC to the soma in the cortex, as both densely and less densely labeled cells were found in all cortical regions.

\section{Auditory and nonauditory cortico-collicular projections}

Our tracing showed a dense and highly lateralized projection from the AC to the three subdivisions of the IC. This is consistent with earlier studies (see Introduction) (for review, see Winer, 2005; Schofield, 2010; Bajo and King, 2013), and serves as a positive control for our exploration of nonauditory corticocollicular connections. In addition to connections from the AC, we demonstrate extensive bilateral cortico-collicular projections from visual, somatosensory, motor, and prefrontal cortices. Although we reveal the full extent of these connections, evidence pointing to the existence of some of these nonauditory corticocollicular connections can be found in an earlier degeneration study in cat by Cooper and Young (1976). Following large lesions of the visual, somatosensory, or motor cortices, these authors reported degenerating fibers in the ipsilateral IC. Although not quantified, their figures suggest that these connections are sparse and mostly confined to areas equivalent to ICD and ICL. Similar findings were reported by RoBards et al. (1976) and RoBards (1979) for somatosensory cortex. More recently, Lesicko et al. (2016), using anterograde tracing, demonstrated connections from somatosensory cortex to the IC in mouse, but they only reported on the presence of labeled terminals in the "neurochemical modules" in ICL (Chernock et al., 2004) that were also recipients of somatosensory inputs from the dorsal column and spinal trigeminal nuclei. In our study, we observed connections distrib- 
uted widely throughout all IC subdivisions, importantly including ICC.

In the case of VC, we observed extensive cortico-collicular connections from both V1 and V2 to the IC. Several functional studies in IC have reported visual modulation of neuronal responses to sounds, and even neuronal responses to visual stimuli alone (Syka and Radil-Weiss, 1973; Tawil et al., 1983; Mascetti and Strozzi, 1988; Porter et al., 2007; Bulkin and Groh, 2012). In the cortices of the IC, these interactions may be explained by direct, but sparse, retinal input (Itaya and Van Hoesen, 1982; Yamauchi and Yamadori, 1982). However, responses to visual stimuli or saccades have also been reported, in ICC, albeit with lower prevalence than in the IC cortices (Porter et al., 2007; Bulkin and Groh, 2012). The longer latency responses observed by Porter et al. (2007) are consistent with effects mediated by indirect pathways via the superior colliculus (Adams, 1980; Coleman and Clerici, 1987), or from the visual cortical input we describe here.

The existence of connections from the VC to the IC may explain recent functional studies in which the BOLD response recorded in the IC using fMRI was shown to be influenced by ablation or optogenetic modulation of the VC (Gao et al., 2015; Leong et al., 2018). Interestingly, the results of these studies implied that input from VC enhanced response gain, whereas input from the $\mathrm{AC}$ reduced gain. Although cortical output projections are considered exclusively excitatory, our finding that projections from cortical regions terminate on both GABAergic and presumed glutamatergic neurons shows that disynaptic circuits in the IC can readily transform cortically mediated excitation to inhibition.

The feedback connections we demonstrate from motor cortex to the IC may be important for two reasons. First, they could be a source of feedback about self-generated sounds, such as vocalizations and sounds that ensue from an animal's interaction with its environment (Schneider and Mooney, 2018; Schneider et al., 2018). Interestingly, neurons in the IC respond prior to vocalization and the IC projects to the neighboring periaqueductal gray (Moore and Goldberg, 1966; Carey and Webster, 1971), a region involved in the control of vocalization (Jürgens, 1994; Pieper and Jürgens, 2003). Motor and somatosensory cortical feedback to the IC could also provide predictive information that enables self-generated sounds to be discounted in the parsing of simultaneous sound sources. That neurons throughout the motor cortex project to the IC suggests that body movements may contribute to this process, perhaps via efference copy, and that such feedback is not limited to the control of vocalization (Schneider and Mooney, 2018). A second reason why motor inputs to the IC are significant is that the IC is one of a network of brain centers involved in escape and other fear responses to aversive sounds. Blockade of inhibition and modulation of NMDA receptors in the IC triggers such behaviors (Brandão et al., 1988; Cardoso et al., 1994; Nobre et al., 2004) and auditory responses in IC are enhanced by unconditioned and conditioned fear evoking stimuli (Brandão et al., 2001). Furthermore, connections from the AC to the IC have been shown to be important in mediating soundevoked defense responses (Xiong et al., 2015).

We know of no existing evidence that the PFC influences responses in the IC. However, neurons in the orbitofrontal regions respond to sound and, via descending connections, influence sound processing in AC (Fritz et al., 2010; Schneider et al., 2014, 2018; Winkowski et al., 2018). These prefrontal cortical regions have a central role in executive function and exert taskdependent influences on sensory processing (Ongür and Price, 2000). Our results demonstrate direct projections from the medial and orbital PFC to the IC and suggest that higher-level cog- nitive functions, including goal-directed behavior, directly affect auditory processing in the midbrain.

Interestingly, whereas neurons projecting to the IC from the visual and auditory cortices displayed a marked ipsilateral dominance, those in the somatosensory, motor, and prefrontal cortices were less lateralized. This might reflect the fact that movement elicited sounds and cognitive mechanisms are not as strongly lateralized as sources of auditory and visual signals.

Extensive descending input to the IC from the cerebral cortex indicates that the IC is more than an integrator of ascending auditory signals. These descending inputs are diverse and include other nonauditory sensory areas as well as nonsensory regions. This diversity of cortical projections suggests that different sensory, motor, and cognitive cortical influences operate at an early stage of processing to influence an animal's perceptual and behavioral responses to sound. We can only speculate about the precise function of this multisystem feedback and how it might mediate the signals required to identify sounds in soundcluttered environments. The mechanisms involved could subserve predictive coding, using priors derived from stimulus history, efference copy to discount self-generated sounds, and the transforms of coordinate frames of reference that enable the senses to be integrated into a coherent representation.

As discussed in the Introduction, there is mounting evidence for the top-down modulation of sensory signals, as well as corticocortical connections mediating interactions between sensory modalities. Our data show that multisystem networks subserving sensory, motor, and executive function extend to subcortical structures. Together, these data demand a revision of current models of sensory perception.

\section{References}

Adams JC (1980) Crossed and descending projections to the inferior colliculus. Neurosci Lett 19:1-5.

Aitkin LM, Dickhaus H, Schult W, Zimmermann M (1978) External nucleus of inferior colliculus: auditory and spinal somatosensory afferents and their interactions. J Neurophysiol 41:837-847.

Aitkin LM, Kenyon CE, Philpott P (1981) The representation of the auditory and somatosensory systems in the external nucleus of the cat inferior colliculus. J Comp Neurol 196:25-40.

Andersen RA, Snyder RL, Merzenich MM (1980) The topographic organization of corticocollicular projections from physiologically identified loci in the AI, AII, and anterior auditory cortical fields of the cat. J Comp Neurol 191:479-494.

Bajo VM, King AJ (2013) Cortical modulation of auditory processing in the midbrain. Front Neural Circuits 6:114.

Bajo VM, Moore DR (2005) Descending projections from the auditory cortex to the inferior colliculus in the gerbil, Meriones unguiculatus. J Comp Neurol 486:101-116.

Bajo VM, Nodal FR, Bizley JK, Moore DR, King AJ (2007) The ferret auditory cortex: descending projections to the inferior colliculus. Cereb Cortex 17:475-491.

Bajo VM, Nodal FR, Moore DR, King AJ (2010) The descending corticocollicular pathway mediates learning-induced auditory plasticity. Nat Neurosci 13:253-260.

Bastos AM, Usrey WM, Adams RA, Mangun GR, Fries P, Friston KJ (2012) Canonical microcircuits for predictive coding. Neuron 76:695-711.

Beyerl BD (1978) Afferent projections to the central nucleus of the inferior colliculus in the rat. Brain Res 145:209-223.

Brandão ML, Tomaz C, Borges PC, Coimbra NC, Bagri A (1988) Defense reaction induced by microinjections of bicuculline into the inferior colliculus. Physiol Behav 44:361-365.

Brandão ML, Coimbra NC, Osaki MY (2001) Changes in the auditoryevoked potentials induced by fear- evoking stimulations. Physiol Behav 72:365-372.

Budinger E, Heil P, Scheich H (2000) Functional organization of auditory cortex in the mongolian gerbil (Meriones unguiculatus): IV. Connections 
with anatomically characterized subcortical structures. Eur J Neurosci 12:2452-2474.

Bulkin DA, Groh JM (2012) Distribution of visual and saccade related information in the monkey inferior colliculus. Front Neural Circuits 6:61.

Cardoso SH, Coimbra NC, Brandão ML (1994) Defensive reactions evoked by activation of NMDA receptors in distinct sites of the inferior colliculus. Behav Brain Res 63:17-24.

Carey CL, Webster DB (1971) Ascending and descending projections of the inferior colliculus in the kangaroo rat (Dipodomys merriami). Brain Behav Evol 4:401-412.

Chernock ML, Larue DT, Winer JA (2004) A periodic network of neurochemical modules in the inferior colliculus. Hear Res 188:12-20.

Coleman JR, Clerici WJ (1987) Sources of projections to subdivisions of the inferior colliculus in the rat. J Comp Neurol 262:215-226.

Coomes DL, Schofield RM, Schofield BR (2005) Unilateral and bilateral projections from cortical cells to the inferior colliculus in guinea pigs. Brain Res 1042:62-72.

Cooper MH, Young PA (1976) Cortical projections to the inferior colliculus of the cat. Exp Neurol 51:488-502.

Coote EJ, Rees A (2008) The distribution of nitric oxide synthase in the inferior colliculus of guinea pig. Neuroscience 154:218-225.

Diamond IT, Jones EG, Powell TP (1969) The projection of the auditory cortex upon the diencephalon and brain stem in the cat. Brain Res 15:305-340.

Feliciano M, Potashner SJ (1995) Evidence for a glutamatergic pathway from the guinea pig auditory cortex to the inferior colliculus. J Neurochem 65:1348-1357.

Friston K (2005) A theory of cortical responses. Philos Trans R Soc Lond B Biol Sci 360:815-836.

Fritz JB, David SV, Radtke-Schuller S, Yin P, Shamma SA (2010) Adaptive, behaviorally gated, persistent encoding of task-relevant auditory information in ferret frontal cortex. Nat Neurosci 13:1011-1019.

Gao EQ, Suga N (2000) Experience-dependent plasticity in the auditory cortex and the inferior colliculus of bats: Role of the corticofugal system. Proc Natl Acad Sci U S A 97:8081-8086.

Gao PP, Zhang JW, Fan SJ, Sanes DH, Wu EX (2015) Auditory midbrain processing is differentially modulated by auditory and visual cortices: an auditory fMRI study. Neuroimage 123:22-32.

Itaya SK, Van Hoesen GW (1982) Retinal innervation of the inferior colliculus in rat and monkey. Brain Res 233:45-52.

Ito T, Malmierca MS (2018) Neurons, connections and microcircuits of the inferior colliculus. In: The mammalian auditory pathways: synaptic organisation and microcircuits (Oliver DL, Cant NB, Fay RR, Popper AN, eds), pp 127-168. New York: Springer.

Ito T, Bishop DC, Oliver DL (2009) Two classes of GABAergic neurons in the inferior colliculus. J Neurosci 29:13860-13869.

Jain R, Shore S (2006) External inferior colliculus integrates trigeminal and acoustic information: unit responses to trigeminal nucleus and acoustic stimulation in the guinea pig. Neurosci Lett 395:71-75.

Jürgens U (1994) The role of the periaqueductal grey in vocal behaviour. Behav Brain Res 62:107-117.

Katz LC, Burkhalter A, Dreyer WJ (1984) Fluorescent latex microspheres as a retrograde neuronal marker for in vivo and in vitro studies of visual cortex. Nature 310:498-500.

Kayser C, Logothetis NK (2007) Do early sensory cortices integrate crossmodal information? Brain Struct Funct 212:121-132.

Leong AT, Dong CM, Gao PP, Chan RW, To A, Sanes DH, Wu EX (2018) Optogenetic auditory fMRI reveals the effects of visual cortical inputs on auditory midbrain response. Sci Rep 8:8736.

Lesicko AM, Hristova TS, Maigler KC, Llano DA (2016) Connectional modularity of top-down and bottom-up multimodal inputs to the lateral cortex of the mouse inferior colliculus. J Neurosci 36:11037-11050.

Mascetti GG, Strozzi L (1988) Visual cells in the inferior colliculus of the cat. Brain Res 442:387-390.

Merchán M, Aguilar LA, Lopez-Poveda EA, Malmierca MS (2005) The inferior colliculus of the rat: quantitative immunocytochemical study of GABA and glycine. Neuroscience 136:907-925.

Moore RY, Goldberg JM (1966) Projections of the inferior colliculus in the monkey. Exp Neurol 14:429-438.

Nobre MJ, Lopes MG, Brandão ML (2004) Defense reaction mediated by
NMDA mechanisms in the inferior colliculus is modulated by GABAergic nigro-collicular pathways. Brain Res 999:124-131.

Oliver DL (2005) Neuronal organisation of the inferior colliculus. In: The inferior colliculus (Winer JA, Schreiner CE, eds), pp 69-114. New York: Springer.

Olthof BMJ, Gartside SE, Rees A (2019) Puncta of neuronal nitric oxide synthase (nNOS) mediate NMDA receptor signaling in the auditory midbrain. J Neurosci 39:876-887.

Ongür D, Price JL (2000) The organization of networks within the orbital and medial prefrontal cortex of rats, monkeys and humans. Cereb Cortex 10:206-219.

Paxinos G, Watson C (1998) The rat brain in stereotaxic coordinates, Ed 4. San Diego: Academic.

Pieper F, Jürgens U (2003) Neuronal activity in the inferior colliculus and bordering structures during vocalization in the squirrel monkey. Brain Res 979:153-164

Porter KK, Metzger RR, Groh JM (2007) Visual- and saccade-related signals in the primate inferior colliculus. Proc Natl Acad Sci USA 104: 17855-17860.

Rao RP, Ballard DH (1999) Predictive coding in the visual cortex: a functional interpretation of some extra-classical receptive-field effects. Nat Neurosci 2:79-87.

Robards MJ (1979) Somatic neurons in the brainstem and neocortex projecting to the external nucleus of the inferior colliculus: an anatomical study in the opossum. J Comp Neurol 184:547-565.

RoBards MJ, Watkins DW 3rd, Masterton RB (1976) An anatomical study of some somesthetic afferents to the intercollicular terminal zone of the midbrain of the opossum. J Comp Neurol 170:499-524.

Saldaña E, Feliciano M, Mugnaini E (1996) Distribution of descending projections from primary auditory neocortex to inferior colliculus mimics the topography of intracollicular projections. J Comp Neurol 371:15-40.

Schindelin J, Arganda-Carreras I, Frise E, Kaynig V, Longair M, Pietzsch T, Preibisch S, Rueden C, Saalfeld S, Schmid B, Tinevez JY, White DJ, Hartenstein V, Eliceiri K, Tomancak P, Cardona A (2012) Fiji: an opensource platform for biological-image analysis. Nat Methods 9:676-682.

Schneider DM, Mooney R (2018) How movement modulates hearing. Annu Rev Neurosci 41:553-572.

Schneider DM, Nelson A, Mooney R (2014) A synaptic and circuit basis for corollary discharge in the auditory cortex. Nature 513:189-194.

Schneider DM, Sundararajan J, Mooney R (2018) A cortical filter that learns to suppress the acoustic consequences of movement. Nature 561:391-395.

Schofield BR (2010) Structural organization of the descending auditory pathway. In: The auditory brain (Rees A, Palmer AR, eds), pp 43-64. Oxford: Oxford UP.

Schofield BR, Schofield RM, Sorensen KA, Motts SD (2007) On the use of retrograde tracers for identification of axon collaterals with multiple fluorescent retrograde tracers. Neuroscience 146:773-783.

Suga N, Ma XF (2003) Multiparametric corticofugal modulation and plasticity in the auditory system. Nat Revs Neurosci 4:783-794.

Syka J, Radil-Weiss T (1973) Acoustical responses of inferior colliculus neurons in rats influenced by sciatic nerve stimulation and light flashes. Int J Neurosci 5:201-206.

Tawil RN, Saadé NE, Bitar M, Jabbur SJ (1983) Polysensory interactions on single neurons of cat inferior colliculus. Brain Res 269:149-152.

Watson RE Jr, Wiegand SJ, Clough RW, Hoffman GE (1986) Use of cryoprotectant to maintain long-term peptide immunoreactivity and tissue morphology. Peptides 7:155-159.

Winer JA (2005) Descending input to the midbrain. In: The inferior colliculus (Winer JA, Schreiner CE, eds), pp 231-247. New York: Springer.

Winer JA, Larue DT, Diehl JJ, Hefti BJ (1998) Auditory cortical projections to the cat inferior colliculus. J Comp Neurol 400:147-174.

Winkowski DE, Nagode DA, Donaldson KJ, Yin P, Shamma SA, Fritz JB, Kanold PO (2018) Orbitofrontal cortex neurons respond to sound and activate primary auditory cortex neurons. Cereb Cortex 28:868-879.

Xiong XR, Liang F, Zingg B, Ji XY, Ibrahim LA, Tao HW, Zhang LI (2015) Auditory cortex controls sound-driven innate defense behaviour through corticofugal projections to inferior colliculus. Nat Commun 6:7224.

Yamauchi K, Yamadori T (1982) Retinal projection to the inferior colliculus in the rat. Cells Tissues Organs 114:355-360. 